\title{
Modeling of beam-induced damage of the LHC tertiary collimators
}

\author{
E. Quaranta, ${ }^{*}$ A. Bertarelli, R. Bruce, F. Carra, ${ }^{\dagger}$ F. Cerutti, A. Lechner, S. Redaelli, and E. Skordis \\ CERN, CH-1211 Geneva 23, Switzerland \\ P. Gradassi \\ A.D.A.M. Applications of Detectors and Accelerators to Medicine, CH-1211 Geneva 23, Switzerland
}

(Received 14 June 2017; published 13 September 2017)

\begin{abstract}
Modern hadron machines with high beam intensity may suffer from material damage in the case of large beam losses and even beam-intercepting devices, such as collimators, can be harmed. A systematic method to evaluate thresholds of damage owing to the impact of high energy particles is therefore crucial for safe operation and for predicting possible limitations in the overall machine performance. For this, a three-step simulation approach is presented, based on tracking simulations followed by calculations of energy deposited in the impacted material and hydrodynamic simulations to predict the thermomechanical effect of the impact. This approach is applied to metallic collimators at the CERN Large Hadron Collider (LHC), which in standard operation intercept halo protons, but risk to be damaged in the case of extraction kicker malfunction. In particular, tertiary collimators protect the aperture bottlenecks, their settings constrain the reach in $\beta^{*}$ and hence the achievable luminosity at the LHC experiments. Our calculated damage levels provide a very important input on how close to the beam these collimators can be operated without risk of damage. The results of this approach have been used already to push further the performance of the present machine. The risk of damage is even higher in the upgraded high-luminosity LHC with higher beam intensity, for which we quantify existing margins before equipment damage for the proposed baseline settings.
\end{abstract}

DOI: 10.1103/PhysRevAccelBeams.20.091002

\section{INTRODUCTION}

Beam intercepting devices, such as collimators, are essential components for accelerators handling high-energy and high-intensity particle beams. Beam losses, either during normal operation or from failures, must be tightly controlled through an effective strategy of collimator settings deployment in order to minimize the risk of damage for all machine equipment. Accelerators with continuously increasing beam intensity, brightness, and stored energies, as planned for the CERN projects LHC injectors upgrade (LIU) [1] and high luminosity LHC [2,3], and other projects such as FAIR (Facility of Antiproton and Ion Research in Europe) [4], spallation neutron sources (e.g. ESS [5]) or future neutrino facilities, push the demands of material robustness for collimators into very challenging grounds.

*elena.quaranta@cern.ch

Also at Politecnico di Milano, Piazza Leonardo da Vinci 32, 20133 Milan, Italy.

Also at Politecnico di Torino, Corso Duca degli Abruzzi, 24, 10129 Turin, Italy.

Published by the American Physical Society under the terms of the Creative Commons Attribution 4.0 International license. Further distribution of this work must maintain attribution to the author(s) and the published article's title, journal citation, and DOI.
The Large Hadron Collider (LHC) has been designed to accelerate proton and lead ion beams to provide collisions with a center-of-mass energy of $14 \mathrm{TeV}$ and $1.15 \mathrm{PeV}$, respectively [6]. The proton operation started at $3.5 \mathrm{TeV}$ in 2010-2011 and the beam energy was increased to $4 \mathrm{TeV}$ in 2012. After two years of shutdown, the accelerator resumed operation in 2015 at $6.5 \mathrm{TeV}$, with the aim of achieving the design value of $7 \mathrm{TeV}$ in the future. The total stored proton beam energy is $362 \mathrm{MJ}$ in the design configuration and 270 MJ have been achieved so far. The protection of the machine from losses of such energetic beam particles, which may induce quenches in the superconducting magnets, is ensured by a multistage collimation system $[6,7]$.

At the LHC, most of the collimators are located in two dedicated cleaning insertions, IR3 and IR7 (Fig. 1). An LHC collimator consists of two movable and parallel jaws, identified conventionally as left and right (see Fig. 2), housed in a tank that is kept under vacuum. The openings of the collimators follow a well-defined transverse hierarchy, as schematically shown in Fig. 3, based on the distance from the beam center measured in units of $\sigma$, i.e. the local betatronic rms beam size at the collimator. The primary collimator (TCP) is positioned closest to the beam, within $5-7 \sigma$, while the secondary collimators (TCSG) are more retracted by usually $1-2 \sigma$. The active part of their jaws is made of a carbon fiber carbon composite that can withstand accidental impact of primary beam for the LHC design 


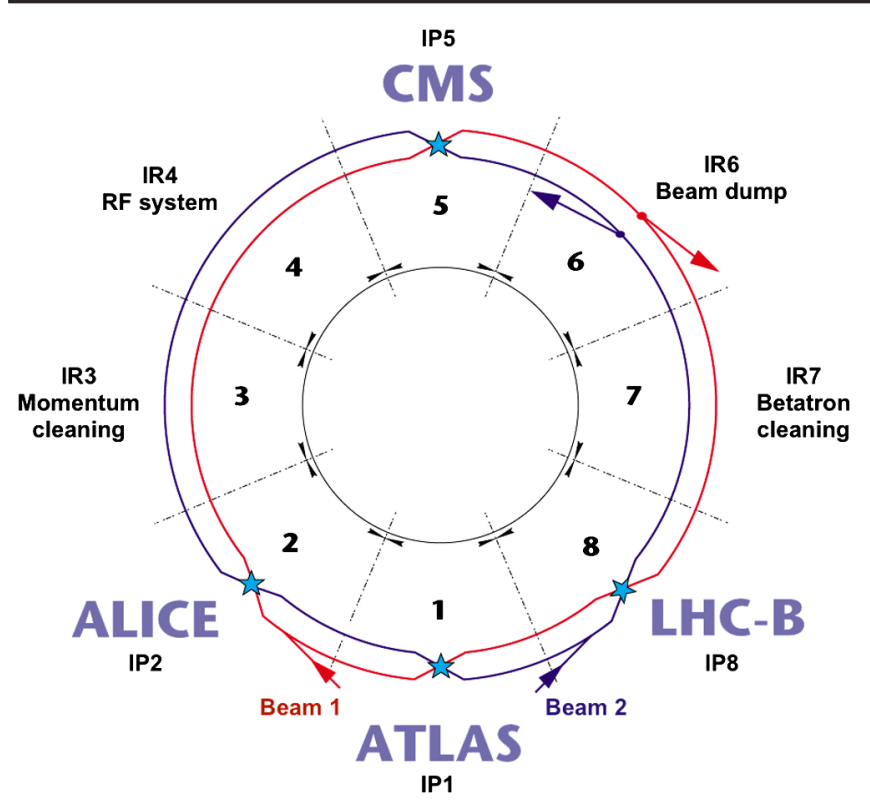

FIG. 1. Schematic layout of the LHC ring.

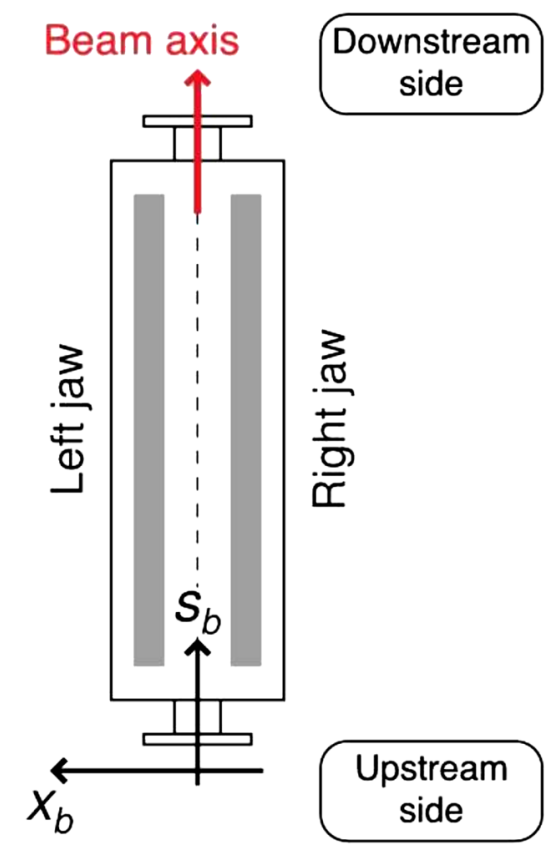

FIG. 2. Collimator coordinate system, as viewed from above [8].

failures [6]. Further away from the beam core, there are absorbers (TCLA), which mainly catch particles that have been scattered out from the previous collimation stages. Finally, tertiary collimators (TCT) provide local protection around the interaction points (IPs). They only intercept a small fraction of beam particles (order of $10^{-3}$ of the inelastic nuclear collisions in the TCP jaws) during standard operation. A heavy tungsten alloy (Inermet-180, or IT-180) is used for these collimators to ensure high

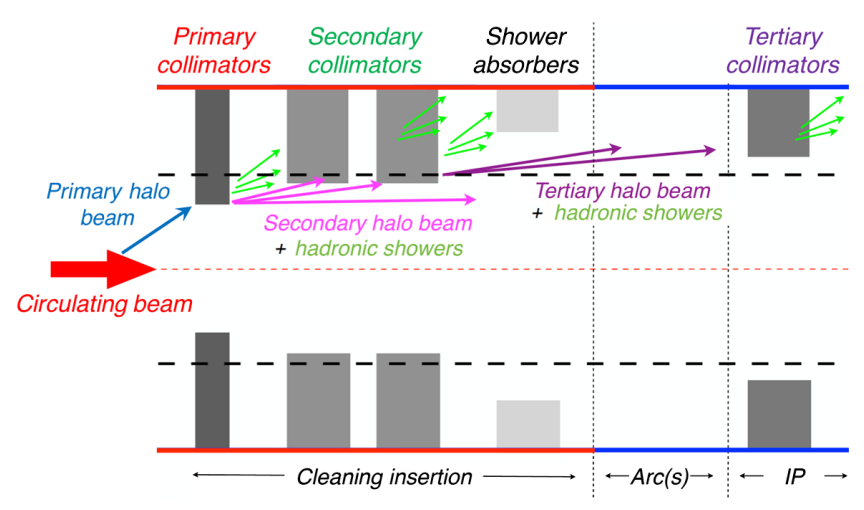

FIG. 3. Scheme of the collimator hierarchy in the LHC.

absorption in favor of robustness. Other graphite-based collimators (namely TCDQ and TCSG6) are used as protection devices and are located along the LHC beam dump lines (IR6).

The first physics run at the LHC proved that the collimation hierarchy constrains the performance in terms of minimum achievable $\beta$-function at the collision points, $\beta^{*}$, determined by the minimum normalized machine aperture that can be protected by the collimators [9]. In the present LHC, the aperture bottlenecks are the inner focusing quadrupoles (triplets) close to the experiments that risk being exposed to local beam losses if not sufficiently protected by the TCTs [9]. The small regular losses during standard operation at the triplets and TCTs do not pose concerns for the material robustness, but protection must be also guaranteed during fast failures, where high beam losses could occur in a short time with consequent damage of the tungsten collimators.

In the future, the HL-LHC upgrade [2] will bring the stored beam energy to almost double the nominal value and will feature brighter beams with the aim of increasing luminosity. Robustness limitations are even more critical in this scenario and are a very important input to the evaluation of the feasibility of the machine design, e.g. for assessing if the collimator settings are compatible with the constraints imposed by machine protection requirements, and could have a direct impact on the material choices for the upgraded collimators [10].

In this article, a simulation method to estimate damage thresholds for beam-intercepting devices is described and applied to various relevant LHC configurations to calculate the limits in terms of protons lost in the collimator jaws below which tungsten TCTs can operate safely without being damaged. Similar approaches to assess the material damage of sensitive accelerator equipment were developed and successfully applied many years ago at the Superconducting Super Collider [11], at the Tevatron Collider [12] as well as at CERN for the SPS and LHC targets, beam dumps and collimators $[13,14]$. The method presented in this paper is based on the latest state-of-the-art tools for improved modeling accuracy. It involves tracking 
simulations of bunches of protons, where a realistic particle impact distribution on the collimators in the studied failure mode provides inputs for calculation of the energy deposited by the beam in the collimator. This in turn is used to study the dynamic response of the collimator material after the beam impact. Damage limits are calculated for the nominal LHC configuration as well as for the future HL-LHC upgrade. The results provide input on how to define a strategy for safe collimator settings during the machine operation. Although the setup is applied in this study to the specific cases of LHC and HL-LHC, the method is generic and can be also applied to other accelerators, present and future, such as the CERN Future Circular Collider (FCC) $[15,16]$.

After presenting the major failure scenarios that may affect the TCTs (Sec. II), the simulation method used for this study is detailed in all its steps (Sec. III) and the definition of the damage limits for collimator materials is given in Sec. IV. Section V presents the simulated scenarios on which this study is focused: for each case, the outcomes are discussed in more detail in Secs. VI-VIII. Section IX presents the results of the damage limit calculations as well as some considerations on the impact of those limits on the machine operation. The interplay between different parameters is studied in Sec. X. Final conclusions are drawn in Sec. XI.

\section{FAILURE SCENARIOS FOR TCTS}

The LHC filling scheme includes an abort gap of about $3 \mu$ s without beam that allow the 15 horizontal extraction kicker magnets (MKDs) to rise up to full field during a standard beam dump. In normal operation mode, the 15 modules have to fire all at the same time, synchronized with the abort gap, in order to correctly extract the beam out of the ring. If any irregularity occurs during this process, single turn (fast) beam losses are generated. Slow losses also occur in standard operation. All beam losses are continuously monitored by the beam loss monitors $[17,18]$ located all around the ring, including at the TCTs. They trigger a beam dump within a few turns if dangerous losses are detected. Slow losses are therefore not in the scope of this paper.

Two major failure scenarios can be identified during a beam abort [19]: one is the simultaneous firing of the 15 kicker modules, but outside the abort gap (asynchronous beam dump). In this case some bunches are affected by the kicker field when it is still rising. A single-module prefire (SMPF), instead, happens when a single kicker spontaneously misfires, out of phase with the abort gap, followed within a short delay by the retriggering of the remaining 14 modules. This case is the most critical one [19]: the slower rise time of the total kick seen by the beam exposes more bunches to smaller kicks. Some miskicked particles cannot reach the dump line and could be, instead, deviated towards the aperture. If not sufficiently in the shadow of the dump protection devices in IR6, the TCTs may be hit by a large fraction of these particles. The tungsten TCT jaws are not designed to intercept such high intensity and could be seriously damaged by the impact. To study realistic damage limits of the TCTs, applicable to LHC operation, we therefore have to model the beam losses during this most critical scenario (SMPF).

\section{THE SIMULATION CHAIN}

Our calculation of damage estimates for beamintercepting devices is based on a three-step simulation setup. Each step is described in more detail in the following subsections. Although we apply the method to impacts on the LHC tertiary collimators during an SMPF, it could equally well be applied to other types of beam loss scenarios.

\section{A. Particle tracking with SIXTRACK}

SIXTRACK is a multiturn six-dimensional symplectic tracking code built for long term beam dynamics studies. It allows computing precisely the trajectories of single charged particles in circular accelerators even with large betatron amplitude and momentum errors [20-23].

A special version of SIXTRACK [24] was developed to model also the interaction with collimators and allows one to estimate the collimation cleaning efficiency in terms of losses generated around the ring as well as of particles reaching the collimator jaws. The dynamics of a large number of particles populating the beam halos can be simulated. When a particle hits a collimator jaw, various physics processes that contribute to the long-range beam losses in the matter-such as ionization energy loss, multiple and single Coulomb scattering, elastic and diffractive nuclear interactions-may occur. In SIXTRACK, such mechanisms are modeled by a Monte Carlo routine described in detail in Refs. [25-27]. Each particle is tracked until either an inelastic interaction occurs at the collimators (the particle is replaced by its secondary products) or it touches the machine aperture, and in both cases it is considered "lost," i.e. removed from the beam. The final output consists of loss maps, containing the spatial and angular coordinates of the lost protons. Simulated loss patterns from SIXTRACK, resulting from regular beam cleaning, have shown a good qualitative and quantitative agreement with LHC data [28].

This setup has been extended to simulate beam failure scenarios at the LHC, like an asynchronous beam dump or an SMPF $[9,29]$, with the whole LHC collimation system in place. The ideal LHC beam dump kicker waveform is used in simulation, assumed to be the same for all the kickers. The retriggering time is $650 \mathrm{~ns}$ with additional $50 \mathrm{~ns}$ between each kicker. This waveform resembles the one recently measured in the LHC [30]. In this study, trains of $25 \mathrm{~ns}$ spaced proton bunches are considered in SIXTRACK. Simulations are performed for both LHC beams at $7 \mathrm{TeV}$ 
with squeezed collision optics (most critical loss scenarios for TCTs).

Each bunch in the train is tracked separately over a few turns along the ring. At the first turn, the simulated particles run normally in the ring. At the second turn, the failure in the dump process is triggered and, when passing the MKDs, each bunch of protons receives a different kick estimated from the measured rise of the kicker magnet field and the bunch position within the train. Miskicked particles continue along a perturbed orbit, with an increased risk of hitting sensitive areas: fractions of several bunches may eventually impact on the TCT jaws. At the third turn, the MKDs have reached their full field and therefore all particles not lost previously are extracted. A file stores the spatial and angular coordinates of the particles lost inside the TCT jaws as a consequence of inelastic interactions, and it provides the input parameters for energy deposition studies.

\section{B. Energy deposition maps with FLUKA}

To estimate the energy deposited inside the impacted material, we use the Monte Carlo program FLUKA [31,32]. Sampling from the positions of the inelastic interactions computed by SIXTRACK, it simulates the interaction and then tracks all created secondary particles, which in turn can interact with the material and create further particles. These hadronic and electromagnetic showers deposit energy in the target material: such 3D energy distribution is the output of the simulation. FLUKA is used routinely to estimate energy deposition at the LHC, and the results have been successfully benchmarked with LHC measurements from beam loss monitors [28,33-35].

The 3D geometry used in FLUKA to model an LHC TCT is shown in Fig. 4. For each 25 ns spaced bunch simulated by SIXTRACK, a map is produced with the energy deposited by the beam in each bin of a scoring grid covering the collimator. In this study, two meshes with different scoring are used: a coarse mesh with bin size of $0.1 \mathrm{~cm}(\mathrm{x}) \times 0.1 \mathrm{~cm}$ (y) $\times 0.42 \mathrm{~cm}(\mathrm{z})$ that covers the whole jaw, and a fine mesh

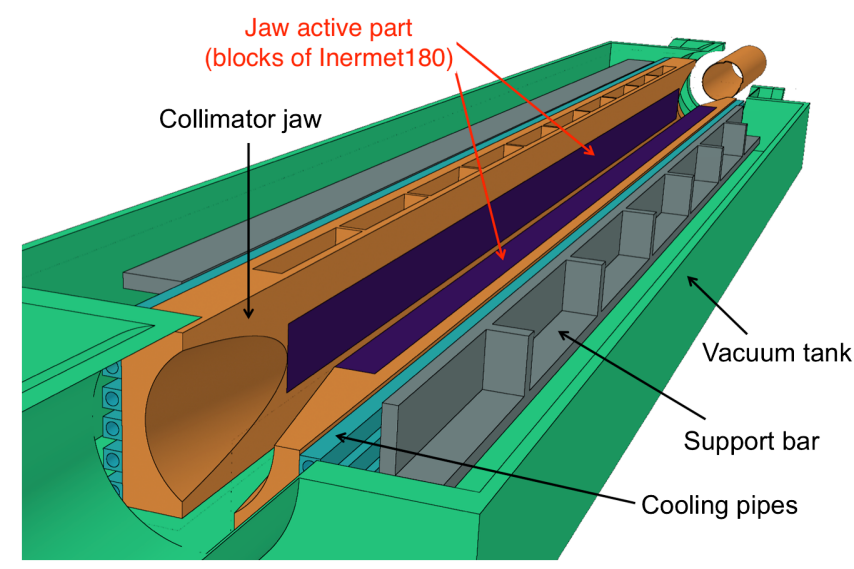

FIG. 4. Geometry of a tertiary collimator as modeled in FLUKA. of $0.05 \mathrm{~cm} \mathrm{(x)} \times 0.05 \mathrm{~cm}(\mathrm{y}) \times 0.5 \mathrm{~cm}(\mathrm{z})$ that covers the impact point. It turned out that the energy deposition profiles look very similar for different bunches. Therefore, for simplicity and increased statistics, a single energy density map has been obtained for any of the studied cases by sampling the initial conditions of the inelastic interactions from the total losses of all bunches.

\section{Thermomechanical studies}

The energy deposited into the jaws, calculated in the previous step, is ultimately turned into heat that leads to temperature increase and to the generation of thermal stresses in the collimator jaws. Depending on the amount and the distribution of the deposited energy and on the time scale of the phenomenon, different effects may result. If the deposited power density is high enough $\left(10^{4} \mathrm{~W} \mathrm{~cm}^{-3}\right.$ or more) and the duration of the interaction is very short (of the order of a few milliseconds or less), dynamic responses are induced, principally because the thermal expansion of the impacted material is partly prevented by its inertia [36]. These effects, often referred to as thermal shocks, generate dynamic stresses, which propagate through the material at the speed of sound. Furthermore, depending on the amount of the deposited energy and on the melting point of the impacted material, the temperature increase induced by the impact may lead to the formation of shock waves, changes of phase, or the ejection of molten material [36]. These complex problems can be treated relying on an advanced class of numerical tools called wave propagation codes or hydrocodes. These are strongly nonlinear, finite element tools, using explicit time-integration schemes, which are particularly suited to deal with very short and intense phenomena implying extensive changes in physical properties.

For our study, mechanical calculations are performed using AUTODYN [37]. In order to derive the evolution of pressure, temperature, and density in the blocks of the jaw, an appropriate equation of state (EOS) must be adopted. In these simulations, a tabular EOS for tungsten has been used for Inermet-180 [38-40]. The behavior of the material beyond the elastic regime is usually accounted for by a strength model, while its structural limits are determined through a convenient failure model: in these simulations the Johnson-Cook strength model [39] and the minimum hydrostatic pressure were adopted [41]. The applied models were compared with data from dedicated experiments at the CERN HiRadMat facility, where a pulsed proton beam impacted collimator materials on a test bench. The results showed a good agreement $[42,43]$.

The FLUKA maps of the energy deposited in the bunches simulated by SIXTRACK are imported into AUTODYN. An energy deposition map is loaded every $25 \mathrm{~ns}$, in accordance to the time structure of the bunch train. Within the $25 \mathrm{~ns}$ granularity, changes of the material properties are taken into account by the EOS of IT-180 as well as the evolution 
of thermomechanical behaviour defined by the strength and failure model. Several dynamic simulations are performed, with varying bunch intensity, until the threshold of a certain damage level is observed in the material.

\section{DEFINITION OF DAMAGE CRITERIA}

In order to quantify whether the material is damaged after beam impacts, we use three levels of damage for different types of structural changes in the tungsten collimators, similarly to Ref. [42].

Below the first damage level (threshold 1) it is assumed that the induced deformation can be almost fully recovered upon removal of the load. The material remains fully elastic or an equivalent plastic strain of no more than $0.2 \%$ is produced. In this case, the expected deformation along the transversal direction is less than a few tens of microns: this damage does not compromise the flatness of the collimator jaw and its cleaning functionality can still be guaranteed. Beyond threshold 1, the material enters in its plastic regime, where it deforms permanently over larger volumes.

Above threshold 2, tungsten fragments are ejected, generating a groove on the jaw surface. In this regime, the cleaning functionality of the collimator risks to be severely jeopardized. However, this might be recovered by moving the jaw along the axis perpendicular to the collimation plane by up to $10 \mathrm{~mm}$ (so-called fifth axis $[6,42])$. In this way, a "fresh," undamaged portion of the jaw is exposed to the beam, replacing the damaged one. Nevertheless, the ejected tungsten fragments can pollute the vacuum tank of the collimator and the beam pipe downstream, which could risk to have effects on operation in terms of vacuum quality and beam losses. If the ejected fragments have to be cleaned, they will result in downtime of the machine.

Finally, if the beam intensity is above threshold 3, the impact leads to severe damage that cannot be recovered even using the fifth axis: the plastic deformation in the material is equal or greater than $2 \%$ with a cylindrical groove of $8 \mathrm{~mm}$ diameter [42]. In these conditions, the integrity of the material is fully compromised and the collimator must be replaced.

As a general design principle, collimator settings and machine configurations are chosen with the aim to minimize the risk of beam losses beyond threshold 1 .

\section{OVERVIEW OF SIMULATED CASES}

The worse case scenario of erratic beam dump caused by the prefiring of the most downstream kicker in the dump line (MKD.A) was reproduced in simulation for both LHC beams. A palette of several cases was simulated to cover a broad range of operational scenarios with different impacts on the TCTs, i.e. various optics versions with varying betatron phase advance between the MKDs and TCTs, and different TCT settings. In this way, comparing several
TABLE I. Collimator retraction settings used in SIXTRACK simulation at $7 \mathrm{TeV}$. The values are expressed in units of standard deviation of the beam, calculated for a normalized emittance of $3.5 \mu \mathrm{m}$ rad. Note that in the table the design settings for TCTs in IP1 and IP5 are listed. In simulations, a scan over a wide range of values has been performed.

\begin{tabular}{lc}
\hline \hline Collimator families & Settings $[\sigma]$ \\
\hline IR7 TCP/TCSG/TCLA & $5.7 / 7.7 / 10.5$ \\
IR3 TCP/TCSG/TCLA & $15 / 18 / 20$ \\
IR6 TCSG/TCDQ & $8.5 / 9$ \\
IR1/5 TCTs & 10.9 \\
IR2/8 TCTs & 30 \\
\hline \hline
\end{tabular}

scenarios and assessing the spread in damage thresholds between them, the results can be more reliably extrapolated to other possible configurations.

The machine configurations considered for this study are the nominal LHC optics with $\beta^{*}=55 \mathrm{~cm}$ and the upgraded HL-LHC optics with $\beta^{*}=15 \mathrm{~cm}$. The collimator openings (Table I) are set according to a $2 \sigma$ retraction between the TCP and TCSG in IR7 [44], where $\sigma$ is the local betatronic beam size at the collimator assuming a nominal normalized emittance of $3.5 \mu \mathrm{m}$ and the design $\beta$-function. In all simulated cases, a normalized emittance of $3.5 \mu \mathrm{m}$ was used, instead of $2.5 \mu \mathrm{m}$, which is the HL-LHC baseline, in order to account for larger tails. Generally, losses at the TCTs are dominated by particles belonging to the beam core, while losses from the tails are few and expected to decrease if a smaller emittance, e.g. $2.5 \mu \mathrm{m}$, is used. Therefore, simulations with a normalized emittance of $3.5 \mu \mathrm{m}$ should provide more pessimistic results than expected for HL-LHC. However, non-Gaussian overpopulated tails could slightly increase the losses over a small range of TCT settings around the opening of the dump protection collimators [45].

SIXTRACK simulations were performed for a perfect machine, without errors on optics, apertures and collimators. However, in operation several errors occur, such as

TABLE II. Betatron phase advances (module expressed in 360 degrees) between MKDs and horizontal TCTs in front of the high-luminosity experiments (ATLAS in IP1 and CMS in IP5). Note that the cases listed in bold in the table corresponds to those simulated for this study.

\begin{tabular}{lccc}
\hline \hline & IP & $\begin{array}{c}\text { Nominal LHC } \\
\beta^{*}=55 \mathrm{~cm}\end{array}$ & $\begin{array}{c}\text { HL-LHC } 1.0 \\
\beta^{*}=15 \mathrm{~cm}\end{array}$ \\
\hline Beam 1 & & & \\
TCTH.4L1.B1 & 1 & $\mathbf{5 6}$ & 209 \\
TCTH.4L5.B1 & 5 & 47 & 245 \\
Beam 2 & & & \\
TCTH.4R1.B2 & 1 & 198 & 140 \\
TCTH.4R5.B2 & 5 & $\mathbf{1 7 6}$ & $\mathbf{1 0 4}$ \\
\hline \hline
\end{tabular}




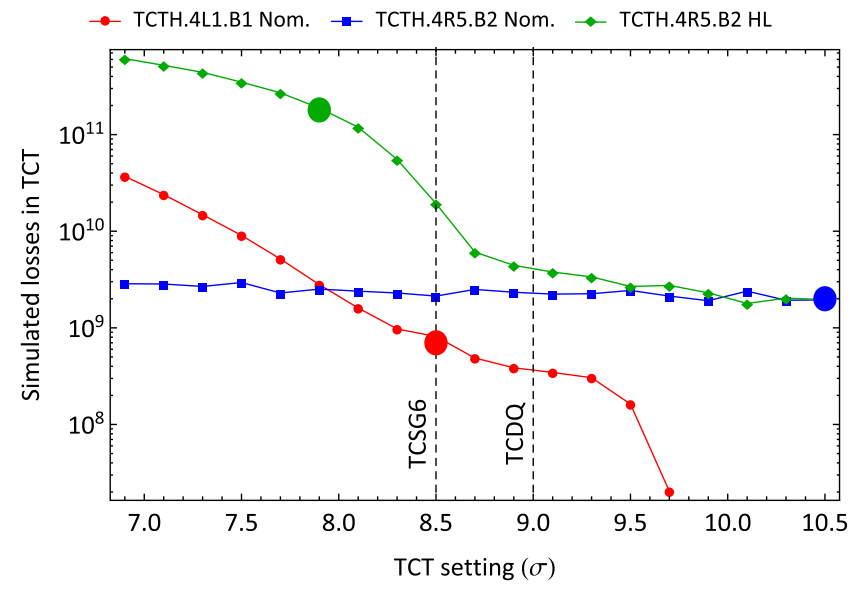

FIG. 5. Losses at TCTs as a function of collimator retraction for different beam optics. Each colored line refers to the most impacted TCT in the three simulated cases. The bigger markers are the scenarios selected for the study.

orbit drifts, collimator misalignments or optics errors. For example, a beta beating of $+20 \%$ would correspond to an increase in beam size by $10 \%$. Such an optics error applied to a collimator at $10 \sigma$ would correspond to a reduction of the effective cut by $1 \sigma$. In simulations, the combination of possible errors that could make the losses worse is accounted for by moving the TCTs closer than the nominal settings (see Table I).

The betatron phase advance from the MKDs to downstream protection collimators (TCSG6 and TCDQ) is by design close to $90^{\circ}$, so that the kicked beam is at its maximum displacement, while the phase advance to the TCTs varies between optics versions, and the different studied TCTs. Phase advance is shown in Table II for the nominal and HL optics, for both beams, and different tertiary collimators. Higher losses are expected if the phase advance from the MKDs is close to $90^{\circ}$ or $270^{\circ}$. TCTH.4L1.B1 appears as the most critical TCT in the nominal optics for Beam 1, while TCTH.4R5.B2 is critical for the HL-LHC optics for Beam 2.

In operation, the TCTs should always be shadowed by the TCDQ. However, imperfections could jeopardize this so that the TCTs may experience large primary proton losses and consequent damage. A first set of SIXTRACK simulations was performed with low statistics $\left(6.4 \times 10^{3}\right.$ macroparticles per bunch) for nominal LHC and HL-LHC optics, scanning over different TCT settings to mimic imperfections. The resulting losses in the TCTs, summed over the simulated bunches and normalized by a bunch population of $1.3 \times 10^{11}$ protons/bunch for nominal LHC and $2.2 \times 10^{11}$ protons/bunch for HL-LHC, are shown in Fig. 5, where we show the most critical TCTs.

Because of the complexity and the time required, three cases were selected to be further studied with higher statistics in the full simulation chain. These cases are summarized in Table III. They were selected in order to have a range of different impact distributions on the TCT jaw.

In Case 1 (blue dot in Fig. 5), protons are intercepted by the TCT regardless of the adopted settings: losses are found even at large TCT settings where the collimator is shadowed by the dump protection devices. It is therefore important to verify that those unavoidable losses, whose cause is discussed in Sec. VI, are not dangerous for the integrity of the collimator jaws. A realistic scenario of losses can occur when TCT is moved at the same settings of TCSG6 [9] (Case 2, red dot), where the TCTs start to be exposed and losses rise with decreasing collimator setting. Finally, a highly pessimistic case is simulated for Case 3 (green dots), where the effective TCT settings are tight and, consequently, the impacts on the jaw are very high. SIXTRACK simulations were repeated for these cases using $6.4 \times 10^{6}$ macroparticles per bunch in order to have a better statistics for the subsequent simulation steps.

In the following, a detailed analysis of each simulated scenario will be presented and the resulting damage thresholds will be discussed.

\section{CASE 1: NOMINAL LHC OPTICS, BEAM 2}

In this case, nearly constant losses occur at the TCTH.4R5.B2 for any settings, even when the TCT is completely shadowed by the dump protection collimators (see Fig. 5). Because of the good phase advance between the MKD and the TCT in this optics (see Table II), it is extremely unlikely that the TCT is directly hit by the miskicked beam. Protons that reach the TCT have instead previously been intercepted by the TCDQ or TCSG6, from where they are scattered back into the beam with a spread in angles and hence large amplitudes. These particles are referred to as secondary protons, to be distinguished from the primary ones that hit directly a TCT without having interacted with any other collimator upstream. Since most secondary protons are scattered to large amplitudes, the

TABLE III. Summary of scenarios selected for simulation studies.

\begin{tabular}{lcccccc}
\hline \hline & $\begin{array}{c}\text { Beam } \\
\text { energy }[\mathrm{TeV}]\end{array}$ & Optics & Beam & $\begin{array}{c}\text { Bunch intensity } \\
{\left[\times 10^{11} \mathrm{p} / \mathrm{b}\right]}\end{array}$ & $\beta^{*}[\mathrm{~cm}]$ & TCT half gap $[\sigma]$ \\
\hline Case 1 & 7 & Nom-LHC & B2 & 1.3 & 55 & 10.5 \\
Case 2 & 7 & Nom-LHC & B1 & 1.3 & 55 & 8.5 \\
Case 3 & 7 & HL-LHC & B2 & 2.2 & 15 & 7.9 \\
\hline \hline
\end{tabular}




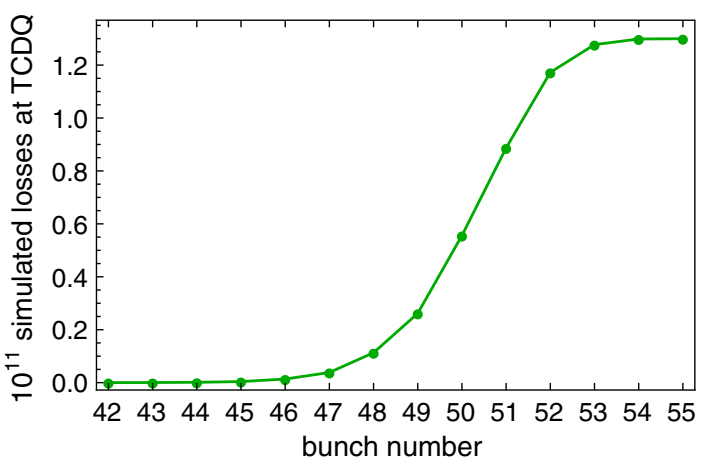

(a) Particles intercepted by TCDQ.

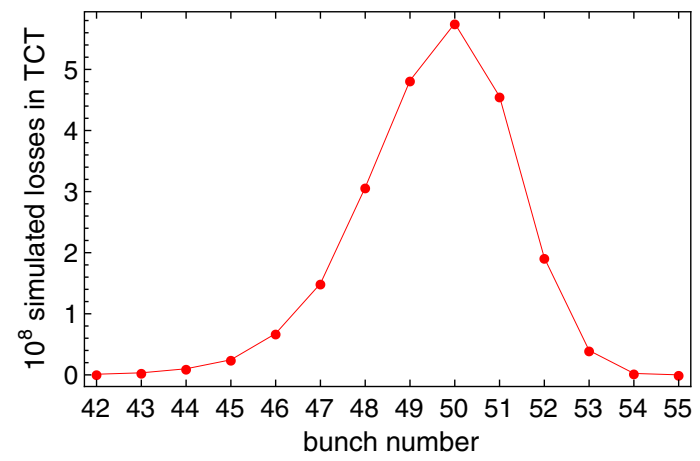

(b) Particles lost in TCTH.4R5.B2.

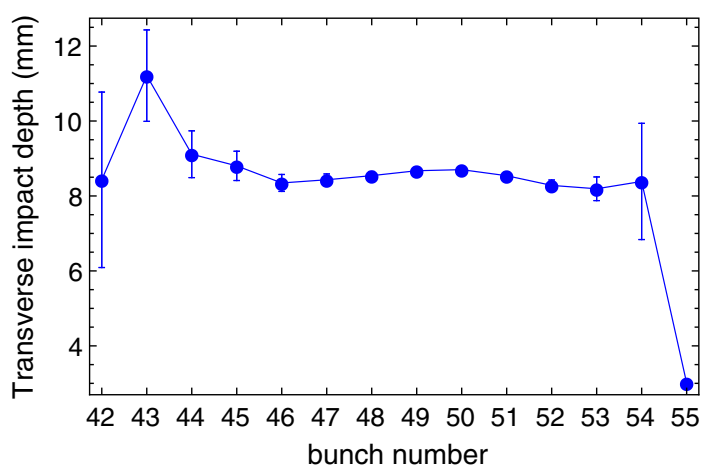

(c) Average impact depth at TCTH.4R5.B2.

FIG. 6. Losses and impact parameter as a function of bunch number for Case 1. A bunch population of $1.3 \times 10^{11} \mathrm{p} / \mathrm{b}$ has been considered for the nominal LHC scenario to scale up the simulated losses on the TCT to a full physics beam.

number of TCT hits is only weakly dependent on the TCT settings in the studied range (see Fig. 5).

In the case of an SMPF, the total kick amplitude increases with time and hence with increasing bunch number. With a bunch spacing of $25 \mathrm{~ns}$, about 140 bunches can be fitted in the time it takes before all MKDs have reached full field, where we define bunch number 1 to pass at the moment of the misfire. However, the first 40 bunches passing by the MKDs receive a very small kick and are dumped in the next turn; particles in bunch numbers greater than 55 are kicked to an amplitude larger than the TCDQ/ TCSG6 aperture and are almost all absorbed in the

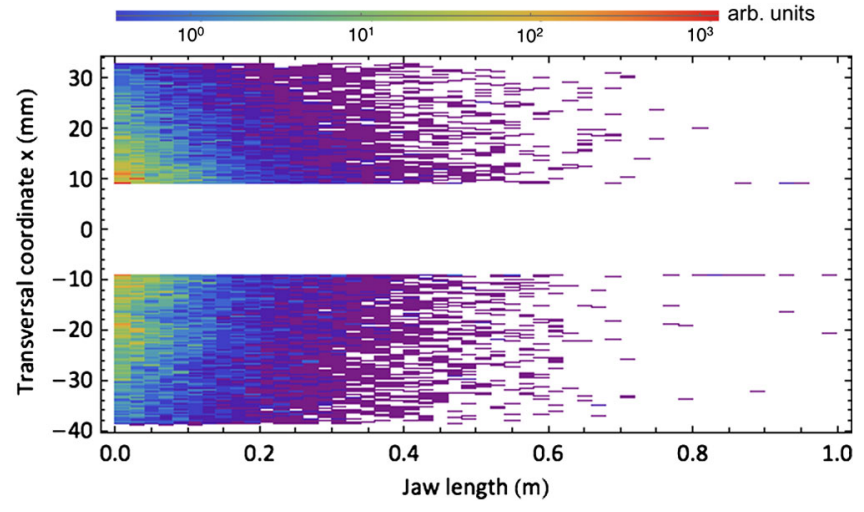

FIG. 7. Density of inelastic nuclear events in the TCTH.4R5.B2 jaws for Case 1. A bin size of $2 \mathrm{~cm}$ in $s$ and $20 \mu \mathrm{m}$ in $x$ is used. Losses are integrated along $y$. The TCT setting (10.5 $\sigma$ is Case 1$)$ is shown with its value in millimeters, i.e. $9.03 \mathrm{~mm}$ from the beam centerline to the edge of the collimator jaw.

protection collimators. Only a few bunches, from 42 to 55, contribute to the losses in the TCT [Fig. 6(a)]. These bunches produce about $98 \%$ of the total number of the impacting protons. Single bunch contribution to losses in TCTH.4R5.B2 is shown in Fig. 6(b). An average impact parameter of about $8 \mathrm{~mm}$ is found over the simulated bunches [Fig. 6(c)]. The transverse distribution of the inelastic interactions along the full jaw length is shown for Case 1 in Fig. 7. The plot shows the transversal coordinate $x$ and the longitudinal one $s$, while the third coordinate $y$ has been integrated. A bin size of $2 \mathrm{~cm}$ is used in $\mathrm{s}$, and $20 \mu \mathrm{m}$ in $\mathrm{x}$. In the left jaw (up), nuclear interactions occur predominantly within the first $20 \mathrm{~mm}$ laterally, while in the right one within $30 \mathrm{~mm}$. Both left and right jaws see the highest nuclear interaction rate in the first $10 \mathrm{~mm}$ laterally. Along the longitudinal coordinate $s$, most of the inelastic events occur within the first $10 \mathrm{~cm}$, which is the nuclear interaction length in the tungsten jaw [46], and this result is in line with the longitudinal profile in Fig. 7.

The coordinates of inelastic interactions within the TCT volume were used in FLUKA for energy deposition calculations, and the resulting map is shown in Fig. 8, sampling from all bunches. Figure 9 shows the peak energy density profile per lost proton along the length of the TCT jaws for Case 1, and the highest peak is reached in the left jaw. The density of inelastic interactions in the two jaws from SIXTRACK simulations (Fig. 7) shows that the loss density in the two jaws is quite similar, although slightly higher for the left one. Therefore, the energy deposited in each jaw from the shower of secondary particles emerging from the other jaw must have contributed to the different energy density.

Thresholds of damage, from the thermomechanical simulations of the structural response of the collimator, are shown in Fig. 10 in comparison with the losses expected at the TCT for the scenario simulated in Case 1 during a single MKD prefiring failure. The blue curve of the protons 


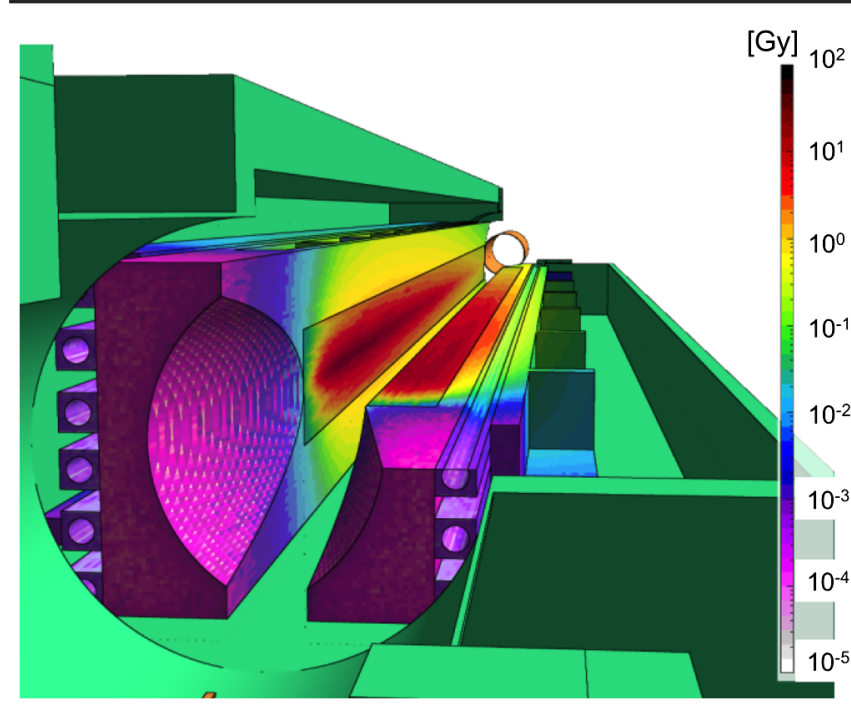

FIG. 8. Map of dose deposited by all the impacting proton bunches on the TCT jaws, obtained by FLUKA simulations, for Case 1.

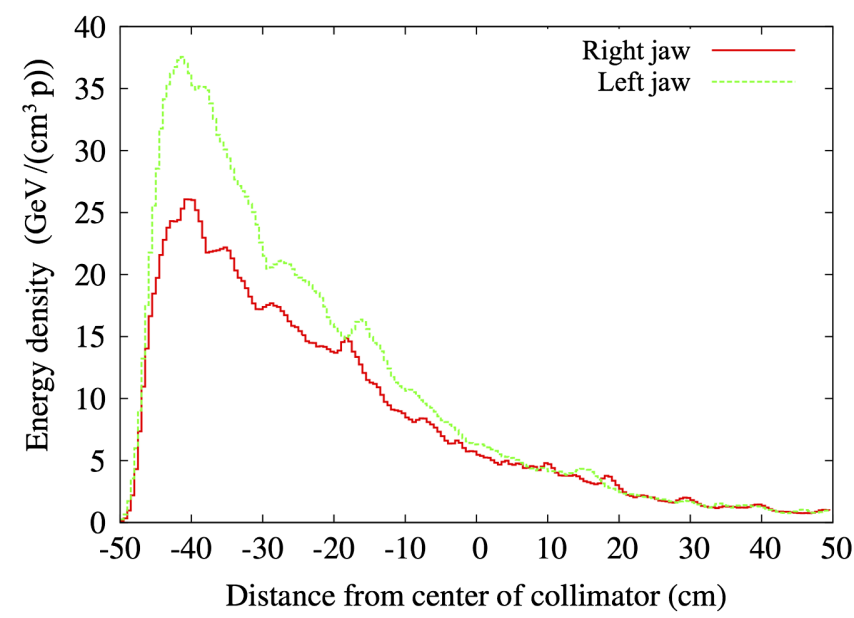

FIG. 9. Maximum energy density per interacting proton over the collimator jaw length, sampled from the losses by all simulated bunches, obtained by FLUKA simulations.

lost in the TCT lies a factor 15 below the value leading to plastic deformation of the material, regardless of the TCT settings used. This means that, for the loss profile per bunch in Fig. 6(b) and the respective impact distribution, we can conclude that the onset of damage in the collimator jaw is expected only with a bunch population 15 times higher.

\section{CASE 2: NOMINAL LHC OPTICS, BEAM 1}

The losses on the TCTH.4L1.B1 for the nominal LHC optics are analyzed in this section. Because of the phase advance of $56^{\circ}$ from the MKDs, this collimator is more exposed to beam losses in the case of dump failure. In particular, for the setting considered in Case 2, the TCSG6 is

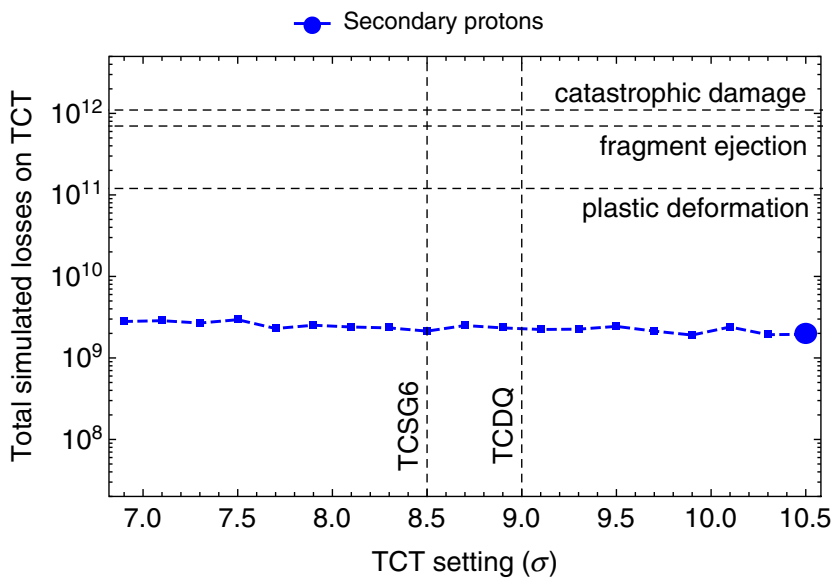

FIG. 10. Losses expected at the TCT in comparison with estimates of damage of the tungsten jaw for Case 1. The losses are estimated assuming a bunch population of $1.3 \times 10^{11} \mathrm{p} / \mathrm{b}$.

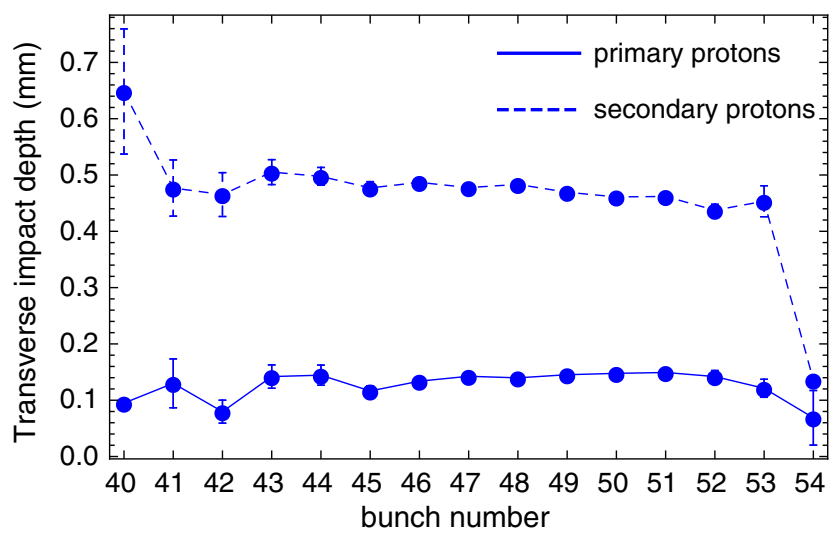

FIG. 11. Average transverse depth of impact from the edge of the collimator jaw given as a function of bunch number for Case 2. A bunch population of $1.3 \times 10^{11} \mathrm{p} / \mathrm{b}$ has been considered.

at the same level as the TCT, therefore, both primary and secondary protons contribute to losses in the collimator. This is clear in Fig. 11 where the impact distribution in the jaw is shown: primary protons interact on average at $100 \mu \mathrm{m}$ from the edge of the collimator, while the secondary particles coming from the upstream collimators show an impact profile which is more spread out inside the jaw, with an average impact parameter of about $500 \mu \mathrm{m}$. The overall distribution of the inelastic interactions along the depth and the length of the jaw is shown in Fig. 12: simulated losses concentrate in one jaw, therefore the opposite jaw is neglected in the plot.

The energy density deposited by the protons in the collimator differs between the left and the right jaw (Fig. 13): the highest peak of lost protons occurs at the left jaw, while the right jaw mainly sees the shower particles which are spread out over the collimator length.

In Fig. 14, we show the losses at the TCT (in red) as a function of the TCT settings, together with the damage 


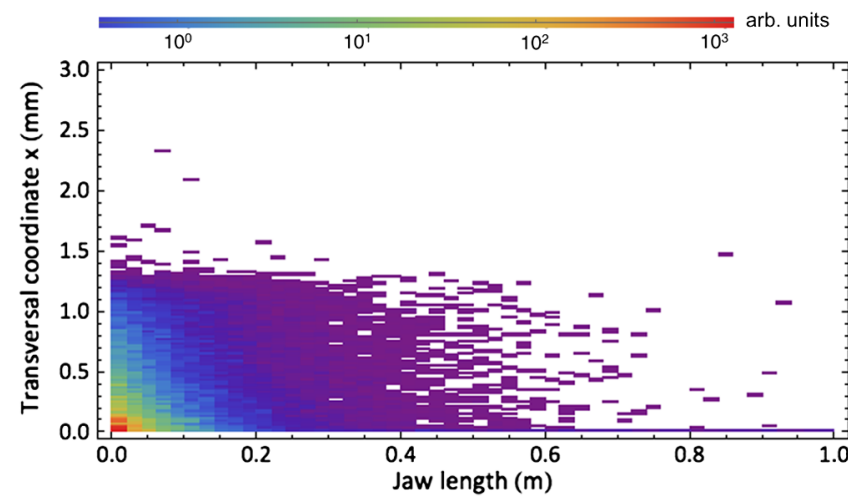

FIG. 12. Density of inelastic interactions in the TCTH.4L1.B1 jaw for Case 2. Simulated losses concentrate in the left jaw, therefore the right one is neglected in the plot. A bin size of $2 \mathrm{~cm}$ in $s$ and $20 \mu \mathrm{m}$ in $x$ is used. Losses are integrated along $y$. In this case, the collimator half gap in millimeters, $7.3 \mathrm{~mm}$, has been already subtracted to the transversal coordinate, so the edge of the jaw is set at $x=0$.

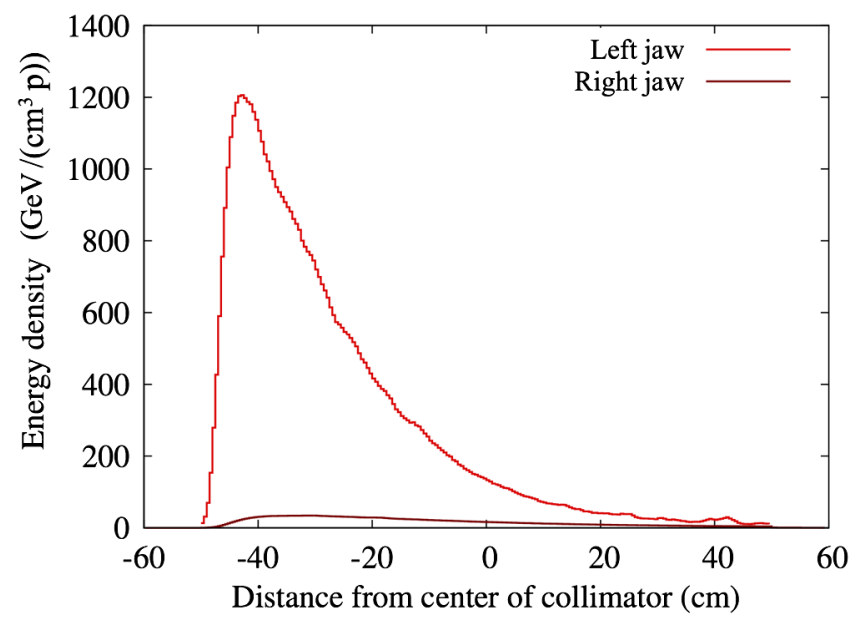

FIG. 13. Peak deposited energy profile along the TCTH.4L1.B1 jaws for Case 2.

limits that were calculated for Case 2 with AUTODYN. In grey, the separated contributions to the losses from primary and secondary protons, solid and dashed curve respectively, are shown. Secondary proton impacts dominate as long as the TCT is in the shadow of the dump protection collimators. Much tighter openings will likely expose the TCT to primary protons that may induce permanent deformation of the jaw and eventually provoke ejection of tungsten fragments from the surface and contaminate the vacuum of the collimator tank (Fig. 15). However, the movement of the jaw along the fifth axis would still allow one to recover the damage generated also at very tight settings.

\section{CASE 3: HL-LHC V1.0 OPTICS, BEAM 2}

A very pessimistic scenario is considered in Case 3: TCTH.4R5.B2 is closed to $7.9 \sigma$, further in with respect to

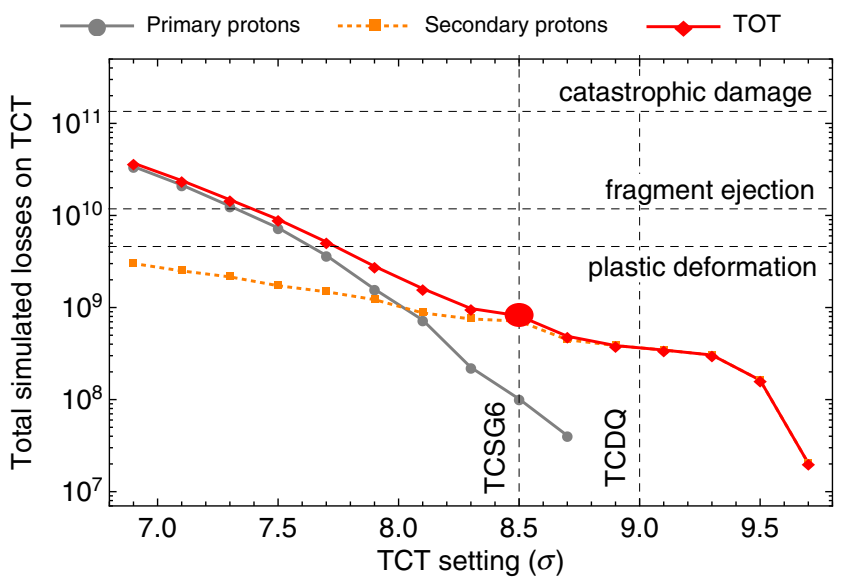

FIG. 14. Simulated losses expected at the TCT compared with the estimates of damage for Case 2. The separated contribution of primary and secondary protons is shown in grey. The losses are estimated assuming a bunch population of $1.3 \times 10^{11} \mathrm{p} / \mathrm{b}$.

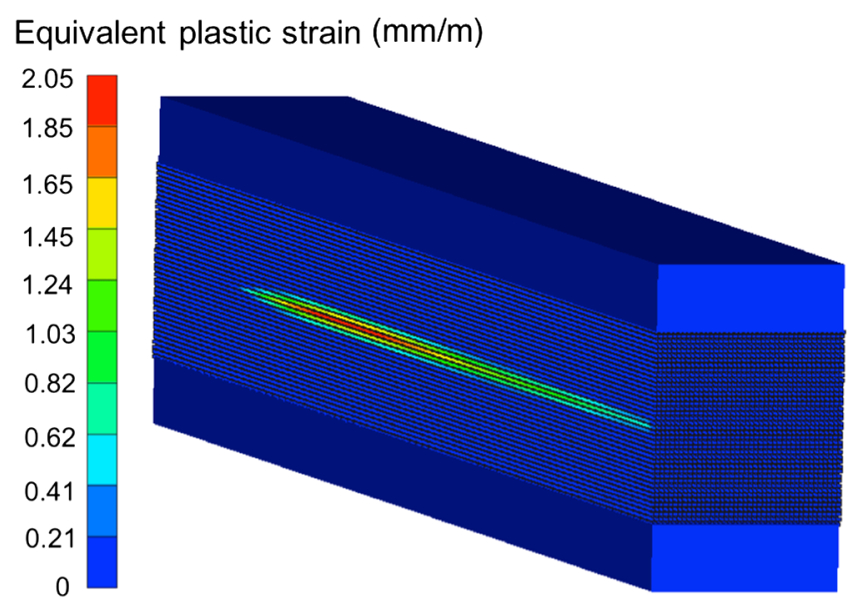

FIG. 15. Plastic deformation in TCTH.4L1.B1 simulated by AUTODYN for Case 2. The picture shows the plastic strain profile corresponding to the onset of permanent damage in the jaw (threshold 1), reached after the impact of the last proton bunch.

the protection collimators of IR6. This is a setting not foreseen for standard operation, but it would represent a catastrophic configuration where large orbit drift or other errors may occur at the time of the dump failure.

In Fig. 16(a) the peak loss in the TCT is slightly shifted to higher bunch numbers compared to previous cases. This is mainly due to the different $\beta$-function at the MKDs for this optics which determines a different amplitude of the kick angle experienced by the bunches. As shown in the loss density profile in Fig. 17, the impacts occur in one jaw because they are dominated by primary protons. In fact, in this case losses from primary beam are concentrated in the first few hundred $\mu \mathrm{m}$ of the jaw, while a tail of secondary particles with lower intensity extends to about $3.5 \mathrm{~mm}$ on average [Fig. 16(b)]. Figure 18 shows that the energy 


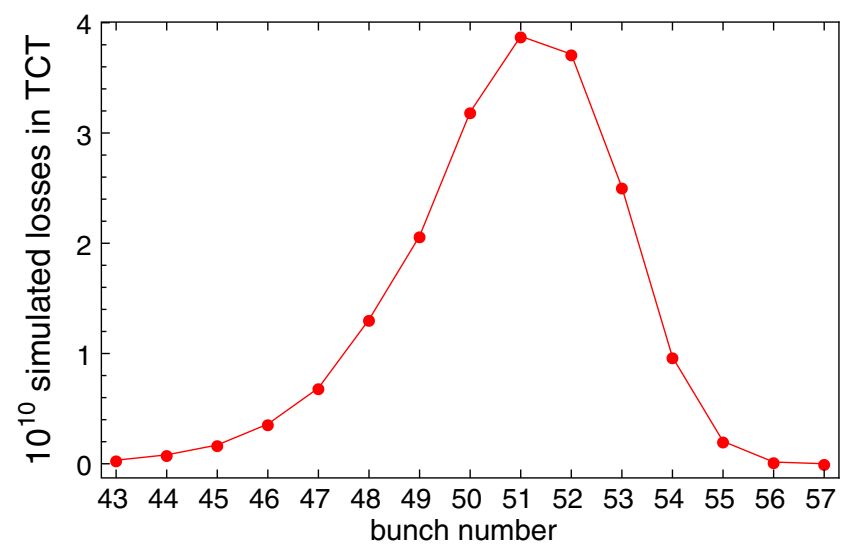

(a) Particles lost in TCTH.4R5.B2.

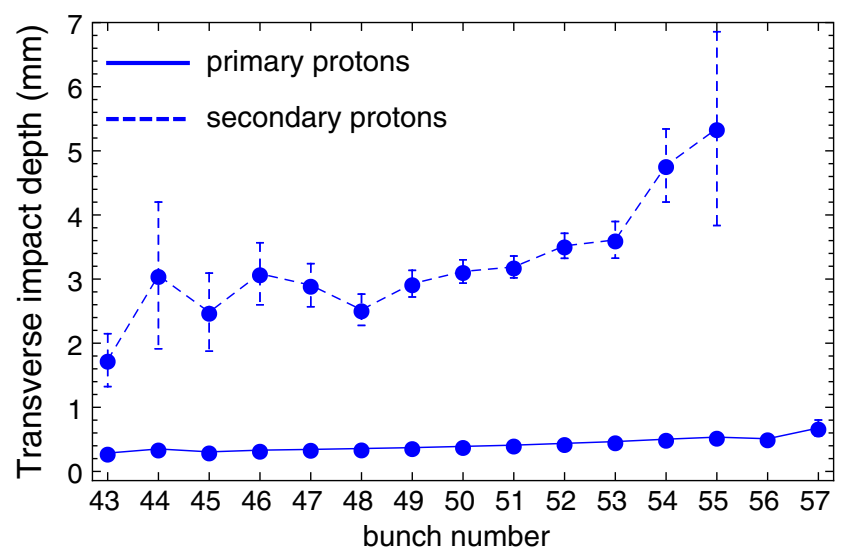

(b) Average impact depth at TCTH.4R5.B2.

FIG. 16. Losses and impact parameter given as a function of bunch number for Case 3 . A bunch population of $2.2 \times 10^{11} \mathrm{p} / \mathrm{b}$ has been considered to estimate the losses on the TCT.

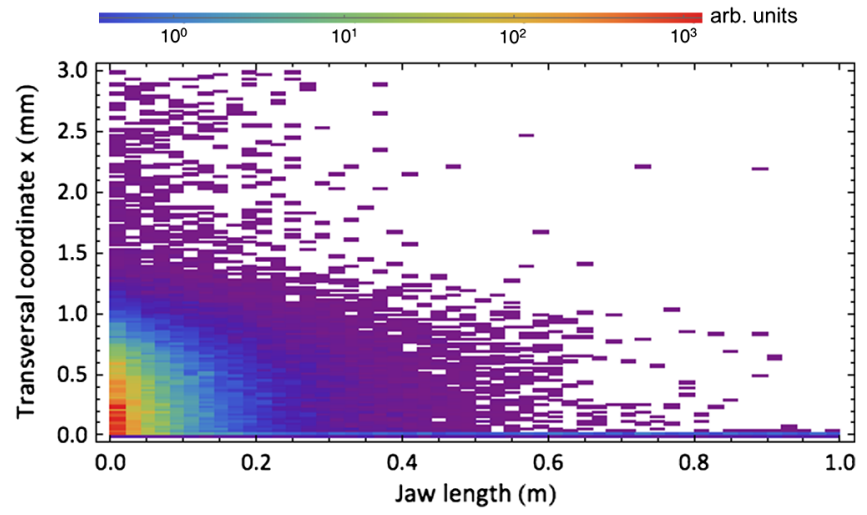

FIG. 17. Density of inelastic interactions in the TCTH.4R5.B2 jaw for Case 3. Simulated losses concentrate in the left jaw, therefore the right one is neglected in the plot. A bin size of $2 \mathrm{~cm}$ in $s$ and $20 \mu \mathrm{m}$ in $x$ is used. Losses are integrated along $y$. In this case, the collimator half gap in millimeters, $11.9 \mathrm{~mm}$, has been already subtracted to the transversal coordinate, so the edge of the jaw is set at $x=0$.

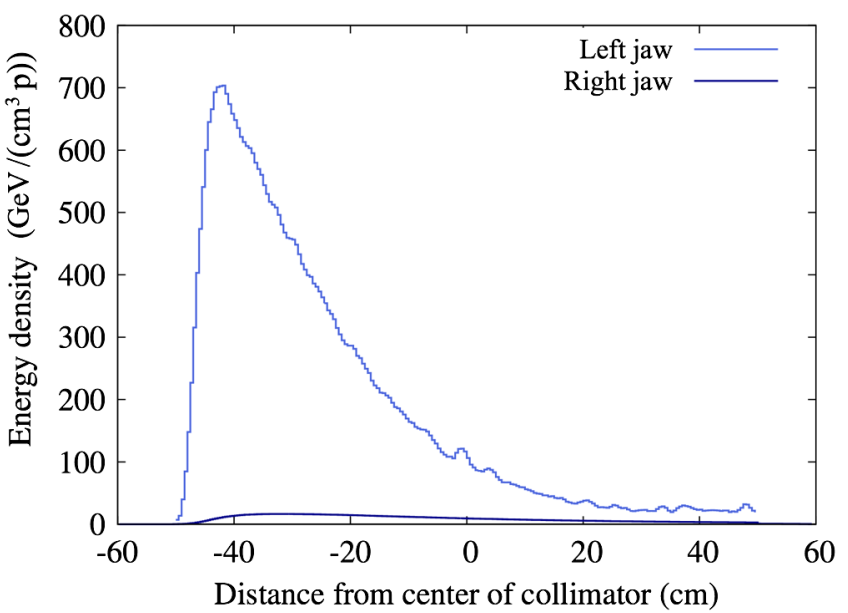

FIG. 18. Peak deposited energy profile along the TCTH.4R5.B2 jaws for Case 3.

density deposited by the protons in the collimator differs between the left and the right jaw and the same explanation adopted for Case 2 applies also here.

The simulation chain was completed and the thresholds of material damage calculated for Case 3. Figure 19 shows the phenomenon of surface spallation when threshold 3 is reached in the TCT jaw. After the impact of the beam against the collimator jaw, a shock wave of compression starts to propagate from the point of the impact to the free surface and the bulk of the jaw. When the wave reaches the free surface, it reflects, changes sign and imposes a tensile stress to the material. In solid mechanics, a spallation process occurs when fragments of material (spalls) are ejected from a body subjected to a tensile stress wave with amplitude higher than the spall strength of the material. The spall strength is defined as the ultimate strength under hydrostatic tension. In the studied scenarios, a simplified failure model was used: first of all, the spall strength is considered constant throughout the simulation, while studies made by [47] showed that it changes as a function

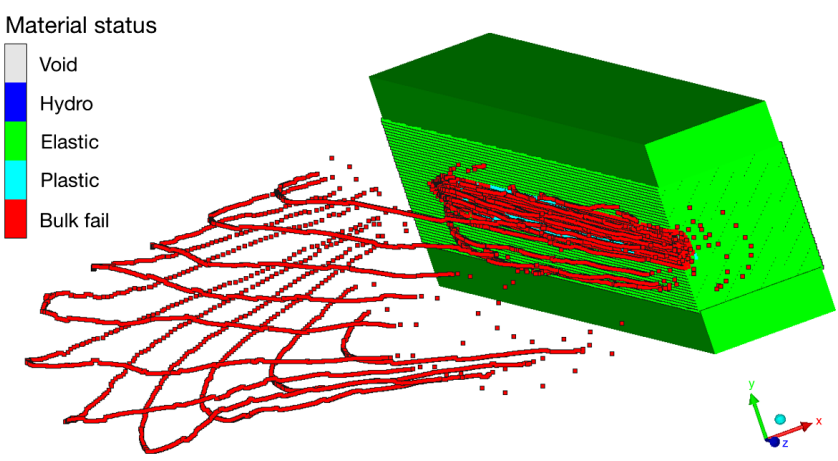

FIG. 19. Status of the TCTH.4R5.B2 after the impact in Case 3: the material of the TCT jaw is deeply damaged and no longer recoverable (threshold 3 ). The traces outside the collimator block represent the ejections of jaw fragments. 


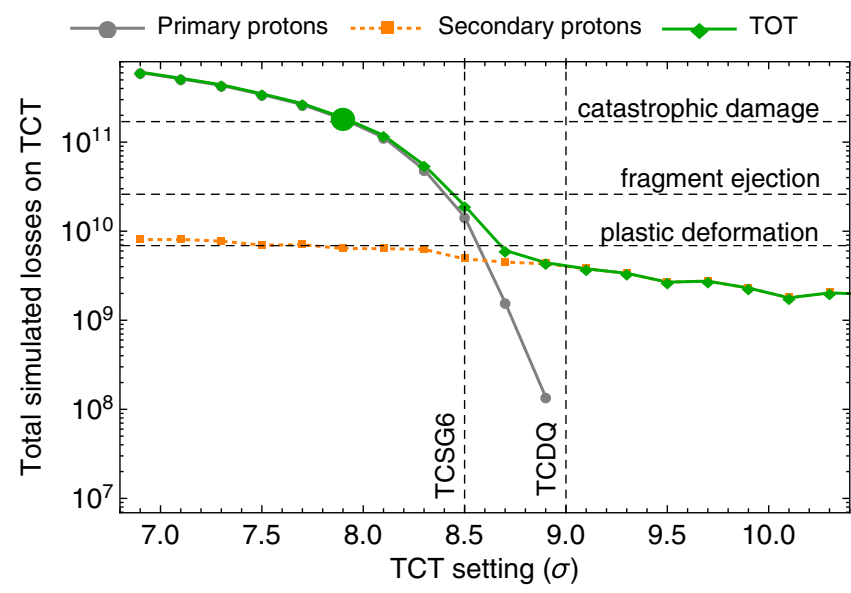

FIG. 20. Simulated losses expected at the TCT compared with the estimates of damage for Case 3 . The contribution of primary and secondary protons is shown in grey. The losses are estimated for a bunch population of $2.2 \times 10^{11} \mathrm{p} / \mathrm{b}$.

of density and flow stress. Additionally, in the simulations the spall strength has been defined as equal to the ultimate strength of a material under uniaxial stress. This is typically a good approximation for brittle materials, for which the Rankine yield criterion apply [48]. However, due to the beam impact, the material experiences a temperature increase above the brittle-to-ductile transition temperature, and exhibits ductile failure. In any case, assuming that the spall strength of a ductile material is equal to the ultimate tensile strength measured with a uniaxial test is a conservative hypothesis, as the first quantity is always higher than the second one [47]. In Inermet-180, the fragments are a mixture of a solid-liquid phase from the low-melting $\mathrm{Cu}$-Ni matrix, melting at $1400^{\circ} \mathrm{C}$, and a solid phase of $\mathrm{W}$, which would melt above $3400^{\circ} \mathrm{C}$. In the strength model defined with AUTODYN, the melting temperature of IT180 was defined as the lowest melting temperature of its constituents, thus that of the $\mathrm{Cu}-\mathrm{Ni}$ matrix. If the temperature after the impact is locally higher than that of the low-melting phase, the material becomes a fluid and it cannot stand a tensile stress state. In the model, this is equivalent to a failure, as the yield stress of the material becomes null. When below the melting temperature, instead, the matrix remains solid and the code considers the ultimate strength value to evaluate the mechanical resistance of the body. As explained, this assumption is conservative.

By looking at Fig. 20, the level of losses due to primary protons estimated for Case 3 would be already so high to irremediably damage the tungsten jaw. However, as pointed out at the beginning of this section, Case 3 represents an extreme scenario which should not be used for normal operation. While, operating at the nominal $10.9 \sigma$ setting for HL-LHC, the losses at the TCT would be dominated by secondary protons, for which the limits are consistently higher (see Fig. 10). Therefore, a strict limit is imposed on the acceptable orbit drifts during HL-LHC operation. If the combined loss in margin between the TCT and the TCDQ would be more than about $2 \sigma$, there is a non-negligible risk for significant damage if an SMPF would occur.

\section{RESULTS}

Table IV shows a summary of the damage thresholds calculated using the three-step method for the study cases presented in this paper.

The number of impacting protons needed to damage the TCT jaw in Case 1 is about a factor 20 higher than in Cases 2 and 3 . The reason of this difference is explained by the transverse impact distribution of primary and secondary protons at the front face of the TCT for the bunch with the largest fraction of particles impinging on the collimator in each simulated case. In Case 1, hits from secondary protons dominate and the impact distribution is quite spread along several millimeters over the width of the jaw [Fig. 21(a)]. The impact profile in Fig. 21(b) shows that for Case 2 a significant contribution to the losses comes also from primary protons which are lost in the first $1 \mathrm{~mm}$ from the edge, with an average of $100 \mu \mathrm{m}$. Secondary losses still dominate but they occur in the first $1.5 \mathrm{~mm}$ : this is because, between the dump line and the TCTH.4L1.B1, there is a betatron collimation insertion in IR7 that cuts off the secondary particles generated in IR6 above a certain amplitude. Finally, primary losses clearly dominates in Case 3 up to $1.5 \mathrm{~mm}$ [Fig. 21(c)], however a tail of secondary particles impacts the TCT up to about $15 \mathrm{~mm}$. By comparing the profiles for the three cases, Case 2 turned out to be the most critical one because the loss profile is very narrow. It can be noticed that the secondary proton distribution in Case 2 is even narrower than that of the primary proton distribution of Case 3 .

In the past simulations, estimates of material damage were calculated by assuming pessimistically that one LHC bunch impacts on the TCT jaw with a fixed impact parameter, without underlying particle tracking studies [42]. The bunch intensity was scaled to identify the value that corresponds to the onset of damage. If we compare threshold $1\left(5 \times 10^{9}\right)$ in Ref. [42] with the value calculated for Case 1, there is more than a factor 20 difference, while the damage limits calculated for Cases 2 and 3 are similar, because of the similar distribution of the protons impacting the TCT. This shows the importance of accounting for the

TABLE IV. Damage limits calculated for the tungsten collimator jaw for the three cases discussed in the paper.

\begin{tabular}{lccc}
\hline \hline & \multicolumn{2}{c}{ Thresholds (number of protons) } \\
Material damage & Case 1 & Case 2 & Case 3 \\
\hline Plastic deformation & $1.2 \times 10^{11}$ & $4.6 \times 10^{9}$ & $6.9 \times 10^{9}$ \\
Fragment ejection & $7 \times 10^{11}$ & $1.8 \times 10^{10}$ & $2.6 \times 10^{10}$ \\
Catastrophic damage & $1.1 \times 10^{12}$ & $1.4 \times 10^{11}$ & $1.7 \times 10^{11}$ \\
\hline \hline
\end{tabular}




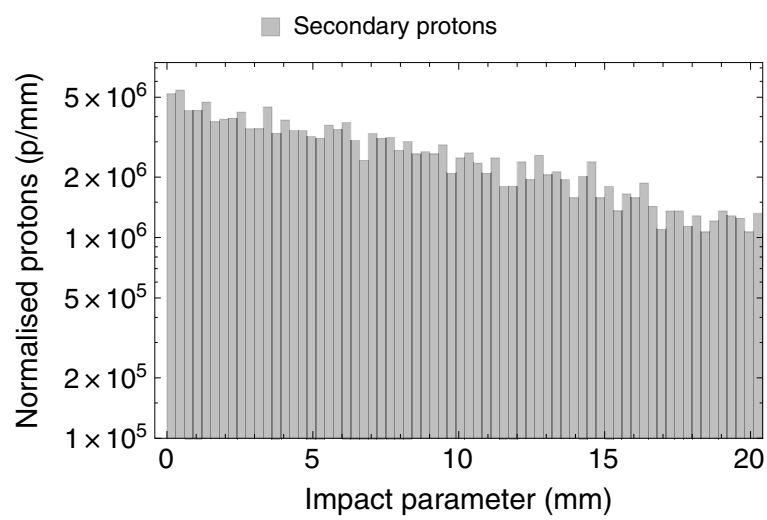

(a) Case 1, TCTH.4R5.B2, bunch 49

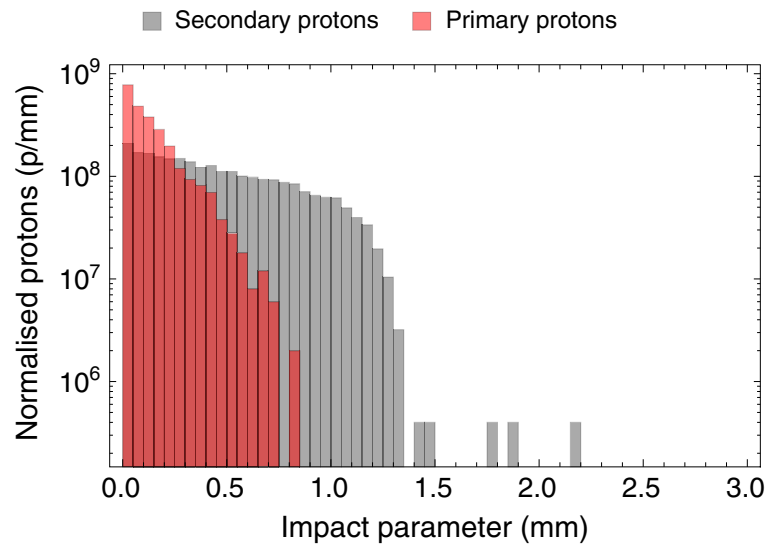

(b) Case 2, TCTH.4L1.B1, bunch 49

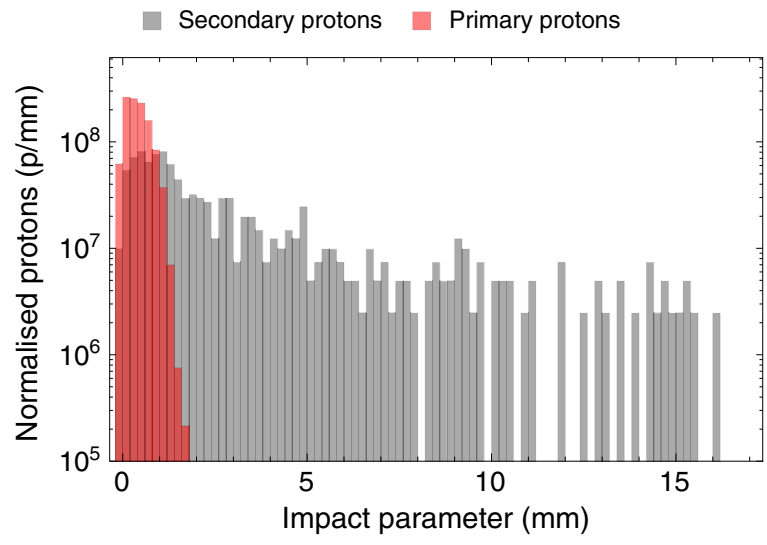

(c) Case 3, TCTH.4R5.B2, bunch 52

FIG. 21. Transverse impact distribution at the face of the TCT for the most impacting bunch in different simulated scenarios. In each case, the histogram bars are normalized to the bin size and to the total number of protons lost. Losses are calculated considering a bunch population of $1.3 \times 10^{11}$ for nominal LHC optics (Cases 1 and 2), and $2.2 \times 10^{11}$ for HL-LHC optics (Case 3).

real impact distribution when determining the damage limit.

Knowing the fraction of each bunch lost at the TCT, one can calculate the "bunch population to damage" (BPD): it is
TABLE V. Bunch population at damage (p/b) calculated for the three cases discussed in the paper, which assume different errors in the TCT settings.

\begin{tabular}{lccc}
\hline \hline TCT setting $[\sigma]$ & Case 1 & Case 2 & Case 3 \\
Material damage & 10.5 & 8.5 & 7.9 \\
\hline Plastic deformation & $7.0 \times 10^{12}$ & $8.4 \times 10^{11}$ & $8.3 \times 10^{9}$ \\
Fragment ejection & $4.0 \times 10^{13}$ & $3.3 \times 10^{12}$ & $3.2 \times 10^{10}$ \\
Catastrophic damage & $6.3 \times 10^{13}$ & $2.5 \times 10^{13}$ & $2.1 \times 10^{11}$ \\
\hline \hline
\end{tabular}

defined as the minimum bunch intensity that would lead to the onset of the different levels of damage. The results for the three simulated cases are listed in Table V. These values can be compared with the achievable bunch population in the machine in each scenario. For Case 1 and Case 2, we compare with $1.3 \times 10^{11}$ protons per bunch, given by injector limitations [49], while for Case 3 we consider the design value for HL-LHC of $2.2 \times 10^{11}$ protons per bunch [2]. In Case 1 one clearly sees that the BPD for threshold 1 is about a factor 50 higher than the nominal case, giving a comfortable safety margin. In this case, the losses are approximately independent of the TCT setting over the considered interval (see Fig. 10), therefore the BPD would be very similar also at different settings. For Case 2 and Case 3, if the TCT moves to tighter settings, larger fractions of each bunch impact the TCT and the impact distribution as well as the BPD could be different. Therefore, one has to keep in mind that the BPD is valid for the specific machine optics and collimator settings simulated in each case. For the scenario in Case 2 with TCT settings at $8.5 \sigma$, for example, the margin of the BPD at plastic deformation from the nominal bunch population is about a factor of 6 . For Case 3, where the TCT are set to $7.9 \sigma$, the BPD for any damage limit is smaller than the HLLHC bunch population $\left(2.2 \times 10^{11}\right)$. This is due to the extreme scenario with very tight settings, which should never be used during standard operation. On the other hand, as previously shown in Fig. 20, for the nominal TCT settings for HL-LHC (10.9 $\sigma)$, only secondary particles are expected to be lost in case of SMPF failure. Therefore, considering a nominal bunch intensity of $2.2 \times 10^{11}$ protons per bunch, the expected losses at the TCT would be about a factor of 5 below threshold 1 for secondary particles (calculated in Case 1). Such a combination of settings and bunch population would thus guarantee a safe machine operation.

In order to ensure the machine to operate in safe conditions with sufficient margins to accommodate possible errors (either in the optics or in the collimator settings), at least around $2 \sigma$ margin is required from the setting where the TCT risks to be damaged [9], with the standard bunch population in each scenario. For instance, the present baseline of HL-LHC with TCT settings in IP1 
and IP5 at $10.9 \sigma$ would guarantee the required margin. On the other hand, reducing $\beta^{*}$ and further tightening the collimator settings must be carefully evaluated. A limitation of bunch population might be imposed unless the TCTs are upgraded with a more robust design. This is part of the HL-LHC upgrade, where new advanced materials are under consideration as replacement of the present tungsten jaw [10]. The losses on TCTs during an SMPF could also potentially be reduced by rematching the optics to improve the phase advance between MKDs and TCTs [50,51], as it was done for the LHC configuration in 2016 [28].

\section{RELATIONS BETWEEN STUDIED PARAMETERS}

In this section, we study various correlations between variables of interest in the simulated cases, starting with the relation between the peak energy deposition, simulated by FLUKA, and the damage limits from AUTODYN. These two variables are shown in Fig. 22, where the symbols of the same color correspond to the value of one damage threshold from Table IV given as a function of the normalized peak energy density in the three cases presented in the paper (Table VI).

The assumptions in Ref. [36] state that the onset of the damage for a given material always occurs at a given total peak energy density, which is the product of the number of impacting protons at damage $y$ (i.e. the damage limit in protons as calculated with AUTODYN) and the peak energy deposition normalized per proton $x$ (as calculated by FLUKA). This product is thus expected to be constant. The points in Fig. 22 are therefore fitted with functions of the type $y=a / x$, one for each damage threshold, where $a$ is a free parameter. Table VII shows the value of $a$ for the different fit curves together with the coefficient of determination $R^{2}$ that gives the goodness of the fit: the closer is $R^{2}$ to 1 , the better is the fit. The product $y \times x$ is close to constant for threshold 1 and the obtained values are

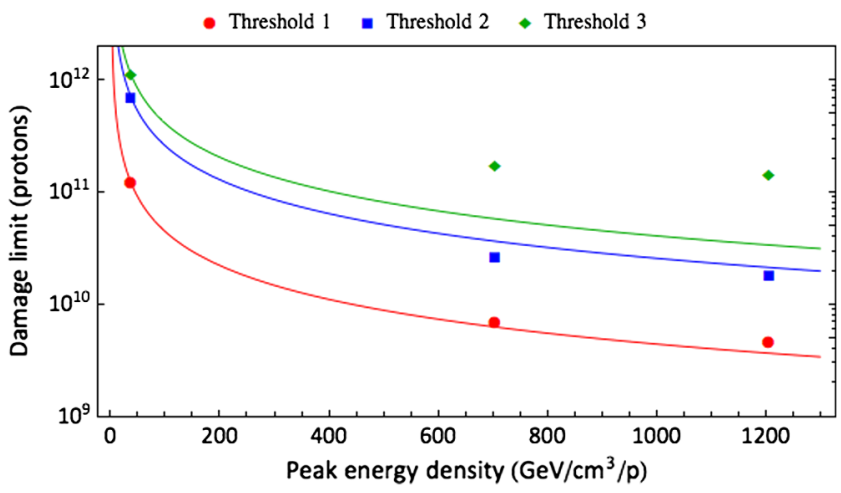

FIG. 22. Correlation between the damage limits of Table IV and the peak energy density per proton for the cases presented in the paper (from left to right: Case 1, Case 3 and Case 2). The lines represent the fit functions $y=a / x$ for each damage threshold.
TABLE VI. Peak energy density deposited per lost proton, calculated by FLUKA, and peak density of inelastic interactions of SIXTRACK macroparticles for the cases discussed in the paper.

\begin{tabular}{lcc}
\hline \hline & $\begin{array}{c}\text { Peak energy density per } \\
\text { proton }\left(\mathrm{GeV} /\left(\mathrm{cm}^{3} / \mathrm{p}\right)\right)\end{array}$ & $\begin{array}{c}\text { Peak probability density of } \\
\text { inelastic interaction }\left(1 / \mathrm{cm}^{3}\right)\end{array}$ \\
\hline Case 1 & 37 & 0.1087 \\
Case 2 & 1206 & 3.0725 \\
Case 3 & 704 & 1.9382 \\
\hline \hline
\end{tabular}

TABLE VII. Fit parameters for the curves in Fig. 22.

\begin{tabular}{lccc}
\hline \hline & Threshold 1 & Threshold 2 & Threshold 3 \\
\hline$a\left(\mathrm{GeV} / \mathrm{cm}^{3}\right)$ & $4.4 \times 10^{12}$ & $2.6 \times 10^{13}$ & $4.1 \times 10^{13}$ \\
$R^{2}$ & 0.999 & 0.999 & 0.981 \\
\hline \hline
\end{tabular}

consistent with the elastic dynamic regime as predicted in Ref. [36]. Threshold 1, indeed, is defined when plasticity occurs, regardless of the size of the plasticized volume, since the local level of strain depends on the specific energy and the coefficient of thermal expansion. Threshold 2, instead, is defined where a local rupture occurs and the relation between energy (or strain) and damage is less straightforward (it also depends on temperature and strain rate). For threshold 3, the nature of the damage is even more complex and dependent on the total energy reached over the defined volume ( $8 \mathrm{~mm}$ diameter cylinder, see IV). Therefore, the fit is less good for thresholds 2 and 3.

Let us consider a proportional relationship between the peak energy density and the peak density of inelastic interactions extracted from SIXTRACK. In the general case, this relationship does not hold, since the peak energy density depends on the convolution of the showers of secondary particles from all nearby events and could be

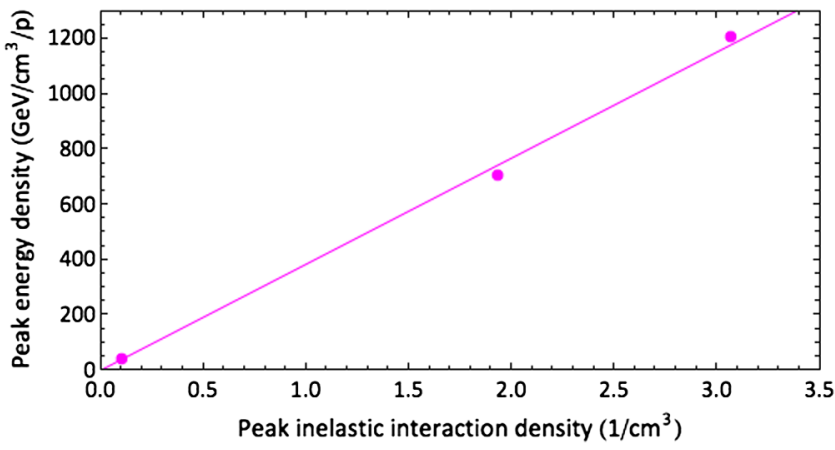

FIG. 23. Correlation between the peak energy density normalized per proton and the peak probability density of inelastic interactions in the TCT for threshold 1 in the simulated cases (from left to right: Case 1, Case 3 and Case 2). The line represents the fit function $x=b z$. 
TABLE VIII. Fit parameters for the curve in Fig. 23.

\begin{tabular}{ll}
\hline \hline Parameter & Value \\
\hline$b(\mathrm{GeV} / \mathrm{p})$ & 384.1 \\
$R^{2}$ & 0.999 \\
\hline \hline
\end{tabular}

highly nonlinear. However, the average energy deposition profile can scale with the number of interactions if the interactions have the same distribution, as assumed in the calculation. To investigate such a relationship, the peak energy density per lost proton, $x$, calculated by FLUKA is shown in Fig. 23 as a function of the peak probability density of inelastic interactions in the TCT, $z$, from SIXTRACK. This is calculated numerically by counting the number of inelastic interactions in all bins, normalizing by the bin volume and the total number of inelastic interactions, and taking the maximum over all bins. Fitting the points with functions $x=b z$, with $b$ free parameter, a good linear fit is found for the three cases studied in this article, as confirmed by the $R^{2}$ value in Table VIII.

By combining the two above assumptions, we find a correlation $y=c / z$ that approximately describes the damage limits (in number of protons) as a function of the peak density of inelastic interactions from SIXTRACK. The fit and the parameter values are shown in Fig. 24 and in Table IX respectively, for threshold 1 . The constant of proportionality $c$ is found to be in the range of $1.3-1.35 \times 10^{10} \mathrm{p} / \mathrm{cm}^{3}$.

In spite of uncertainties in the underlying assumptions, the fit for threshold 1 describes well the three simulated cases. In future studies, a first guess of the onset of damage of the TCT jaw in different scenarios could be derived from the fit, using SIXTRACK simulations. For example, the fit can be useful to identify and down-select scenarios of interest to be further studied in detail. However, we stress that for reliable damage limits, it is important to perform the full simulation chain.

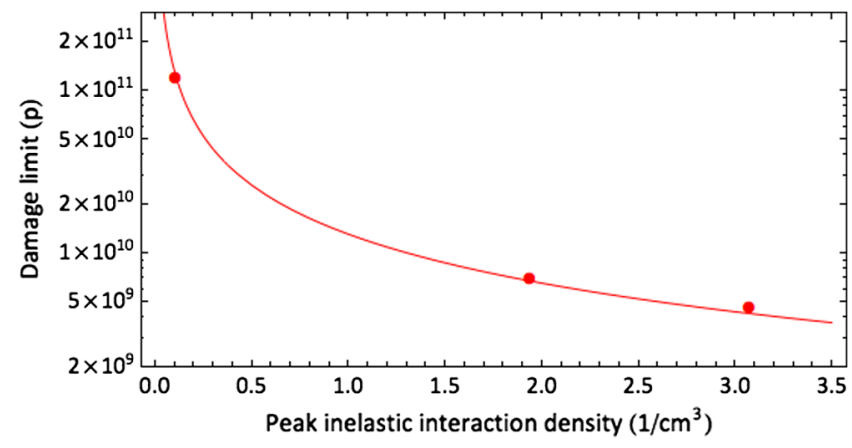

FIG. 24. Correlation between the peak probability density of inelastic interactions in the TCT and the damage limits calculated for threshold 1 in the simulated cases (from left to right: Case 1, Case 3 and Case 2). The line represents the fit function $y=c / z$.
TABLE IX. Fit parameters for the curve in Fig. 24.

\begin{tabular}{lc}
\hline \hline Parameter & Value \\
\hline$c\left(\mathrm{p} / \mathrm{cm}^{3}\right)$ & $1.31 \times 10^{10}$ \\
$R^{2}$ & 0.999 \\
\hline \hline
\end{tabular}

\section{CONCLUSIONS}

In high-energy hadron machines, it is crucial to have a reliable way of estimating the onset of beam-induced damage to accelerator components, such as collimators, to ensure safe operation.

In this paper, we have exploited a method to calculate such damage thresholds using a three-step simulation approach, where previously the individual steps have been successfully benchmarked with experimental data. This method consists of tracking studies to determine beam impact conditions for design failure cases, energy deposition studies, and thermomechanical analysis of the dynamic response of the material to pressure waves propagation inside its structure.

We have applied the method to study possible damage of tertiary collimators in the LHC, if they are hit by miskicked beam during a beam extraction failure. Three possible scenarios with different optics and beam conditions have been investigated. The results have already been used to define a strategy of appropriate collimator settings to deploy in LHC operation, and also to provide crucial inputs for the configuration of the future HL-LHC.

Some correlations have been found between the studied parameters, which could be used for a first approximated estimate of the onset of damage, e.g. to help selecting critical scenarios that are relevant for further studies. However, the full simulation chain must be used to provide more reliable estimates of the limits, in particular for the cases of catastrophic damage that would imply the collimator replacement.

The presented method was applied to the specific case of tungsten-based collimator jaw, according to the present design of tertiary collimators in the LHC. In the future, similar studies could be repeated for different materials that are under consideration for the HL-LHC collimation upgrade.

\section{ACKNOWLEDGMENTS}

We would like to thank CERN colleagues from FLUKA team, Mechanical and Material Engineering group, and the Accelerator Physics group, in particular the LHC Collimation Project team, for the helpful inputs and many fruitful discussions that made the realization of this work possible. We also acknowledge the EuCARD-2 project (Grant Agreement No. 312453) and the High Luminosity LHC project, which funded the presented research work. 
[1] M. Meddahi et al., LHC injectors upgrade (LIU) project at CERN, in Proceedings of the 6th International Particle Accelerator Conference, Richmond, VA (JACoW, Richmond, 2015), Vol. 6, pp. 3915-3918.

[2] I. B. Alonso et al., HiLumi LHC Technical Design Report No. CERN-ACC-2015-0140, 2015.

[3] O. Brüning and L. Rossi, The high luminosity Large Hadron Collider, edited by O. Brüning and G. Arduini (World Scientific Publishing, Singapore, 2015).

[4] FAIR-Baseline technical report, Executive summary, edited by $\mathrm{H}$. Gutbrod et al. (Gesellschaft für Schwerionenforschung mbH, Darmstadt, Germany, 2006), Vol. 1.

[5] S. Peggs et al., ESS Technical Design Report No. ESS-doc274-v15, 2013.

[6] O. Brüning, LHC design report Vol. 1: The LHC main ring, 2004.

[7] S. Redaelli et al., LHC Aperture Measurements, in Proceedings of the International Particle Accelerator Conference, Kyoto, Japan (ICR, Kyoto, 2010), C100523.

[8] G. Valentino, Ph.D. thesis, University of Malta, 2013 [Report No. CERN-ACC-2014-0062].

[9] R. Bruce et al., Calculations of safe collimator settings and $\beta^{*}$ at the CERN Large Hadron Collider, Phys. Rev. ST Accel. Beams 18, 061001 (2016).

[10] E. Quaranta et al., Towards optimum material choices for the HL-LHC collimator upgrade, in Proceedings of the International Particle Accelerator Conference (IPAC'16), Busan, Korea, 2016 (JACoW, Busan, 2016), Vol. 7, pp. 2498-2501.

[11] D. C. Wilson et al., Hydrodynamic calculations of 20-TeV beam interactions with the SSC beam dump, in Proceedings of the 15th Particle Accelerator Conference, PAC1993, Washington, DC, 1993 (IEEE, New York, 1993), Vol. 4, pp. 3090-3092.

[12] A. I. Drozhdin et al., Report No. FERMILAB-FN-751, 2014.

[13] N. A. Tahir, R. Schmidt, M. Brugger, R. Assmann, A. V. Shutov, I. V. Lomonosov, A. R. Piriz, D. H. H. Hoffmann, C. Deutsch, and V.E. Fortov, The CERN Super Proton Synchrotron as a tool to study high energy density physics, New J. Phys. 10, 073028 (2008).

[14] N. A. Tahir, J. B. Sancho, A. Shutov, R. Schmidt, and A. R. Piriz, Impact of high energy high intensity proton beams on targets: Case studies for Super Proton Synchrotron and Large Hadron Collider, Phys. Rev. ST Accel. Beams 15, 051003 (2012).

[15] A. Ball et al., CERN Technical Report No. FCC-ACCSPC-0001, Geneva, Switzerland, 2014.

[16] M. Fiascaris et al., First design of proton collimation system for $50 \mathrm{TeV}$ FCC-HH, in Proceedings of the International Particle Accelerator Conference (IPAC'16), Busan, Korea, 2016 (Ref. [10]), Vol. 7, pp. 2423-2426.

[17] B. Dehning et al., The LHC beam loss measurement system, in Proceedings of the 22nd Particle Accelerator Conference, PAC-2007, Albuquerque, NM (IEEE, New York, 2007).

[18] E. B. Holzer et al., Report No. CERN-AB-2006-009, 2005.

[19] R. W. Assmann et al., The consequences of abnormal beam dump actions on the LHC collimation system, LHC Project Note 293, CERN, 2002.

[20] F. Schmidt, Report No. CERN/SL/94-56-AP, 1994.
[21] SIXTRACK, http://sixtrack.web.cern.ch/SixTrack/.

[22] R. De Maria et al., Recent developments and future plans for SIXTRACK, in Proceedings of the 4th International Particle Accelerator Conference, IPAC-2013, Shanghai, China, 2013 (JACoW, Shanghai, China, 2013).

[23] A. Mereghetti et al., SIXTRACK for cleaning studies: 2017 updates, in Proceedings of the 8th International Particle Accelerator Conference, IPAC-2017, Copenhagen, Denmark (JACoW, Copenhagen, Denmark, 2017).

[24] G. Robert-Demolaize et al., A new version of SIXTRACK with collimation and aperture interface, in Proceedings of the 21st Particle Accelerator Conference, Knoxville, TN, 2005 (IEEE, Piscataway, NJ, 2005).

[25] N. Catalan Lasheras, Report No. CERN-THESIS-2000019, Zaragoza, 1998.

[26] G. Robert-Démolaize, Report No. CERN-THESIS-2006069, Joseph Fourier University, Grenoble, 2006.

[27] C. Tambasco, Report No. CERN-THESIS-2014-014, UniversitàLa Sapienza, Roma, Italy, 2014.

[28] R. Bruce et al., Simulations and measurements of beam loss patterns at the CERN Large Hadron Collider, Phys. Rev. ST Accel. Beams 17, 081004 (2014).

[29] L. Lari et al., Accelerator physics studies on the effects from an asynchronous beam dump onto the LHC experimental region collimators, in Proceedings of the 3rd International Particle Accelerator Conference, New Orleans, LA, 2012 (IEEE, Piscataway, NJ, 2012), C1205201, pp. 547-549.

[30] M. Fraser et al., A beam-based measurement of the LHC beam dump kicker waveform, in Proceedings of the International Particle Accelerator Conference (IPAC'16), Busan, Korea, 2016 (Ref. [10]), Vol. 7, pp. 3911-3913.

[31] A. Ferrari et al., Report No. CERN-2005-010, 2005.

[32] T. T. Böhlen, F. Cerutti, M. P. W. Chin, A. Fassò, A. Ferrari, P. G. Ortega, A. Mairani, P. R. Sala, G. Smirnov, and V. Vlachoudis, The FLUKA code: Developments and challenges for high energy and medical applications, Nucl. Data Sheets 120, 211 (2014).

[33] B. Auchmann et al., Testing beam-induced quench levels of LHC superconducting magnets, Phys. Rev. ST Accel. Beams 18, 061002 (2015).

[34] V. Boccone et al., Beam-machine. Interaction at the CERN LHC, Nucl. Data Sheets 120, 215 (2014).

[35] E. Skordis et al., Impact of beam losses in the LHC collimation regions, in Proceedings of the 6th International Particle Accelerator Conference, Richmond, VA (Ref. [1]), Vol. 6, pp. 2116-2119.

[36] A. Bertarelli, Beam-induced damage mechanisms and their calculation, http://dx.doi.org/10.5170/CERN-2016002, Vol. 159, pp. 159-227.

[37] ANSYS AUTODYN User Manual, release 13.0, 2010.

[38] A. Bertarelli et al., Limits for beam-induced damage: Reckless or too cautious?, in Proceedings of the Chamonix 2011 Workshop on LHC Performance, Chamonix, France (CERn, Geneva, 2011), pp. 183-188 [Report No. CERN2011-005].

[39] M. Scapin et al., Mechanical characterization and modeling of the heavy tungsten alloy IT180, Int. J. Refract. Metals Hard Mater. 50, 258 (2015). 
[40] A. Bertarelli et al., An experiment to test advanced materials impacted by intense proton pulses at CERN HiRadMat facility, Nucl. Instrum. Methods Phys. Res., Sect. B 308, 88 (2013).

[41] A. Bertarelli et al., Behaviour of advanced materials impacted by high energy particle beams, International Symposium on Dynamic Deformation and Fracture of Advanced Materials, Loughborough, United Kingdom, 2013.

[42] A. Bertarelli et al., Updated robustness limits for collimator material, in Proceedings of the LHC Machine Protection Workshop, Annecy, France, edited by M. Jonker (CERn, Geneva, 2013), pp. 108-112 [Report No. CERN-ACC2014-0041].

[43] M. Cauchi et al., High energy beam impact tests on a LHC tertiary collimator at the CERN high-radiation to materials facility, Phys. Rev. ST Accel. Beams 17, 021004 (2014).

[44] R. Bruce et al., Report No. CERN-ACC-2014-0044, 2014.

[45] R. Bruce et al., Report No. CERN-ACC-2016-0328, 2016.

[46] E. Quaranta et al., Collimation cleaning at the LHC with advanced secondary collimator materials, in Proceedings of the 6th International Particle Accelerator Conference, Richmond, VA (Ref. [1]), Vol. 6, pp. 2068-2071.

[47] D. E. Grady, The spall strength of condensed matter, J. Mech. Phys. Solids 36, 284 (1987).

[48] W. Rankine, On the stability of loose earth, Phil. Trans. R. Soc. London 147, 9 (1857).

[49] Y. Papaphilippou et al., Operational beams for the LHC, in Chamonix 2014: LHC Performance Workshop, Chamonix, France, 2014, edited by M. Draper (CERn, Geneva, 2014), pp. 80-84.

[50] R. Bruce et al., 2016 LHC configuration: Can we get to $\beta^{*}=40 \mathrm{~cm}$ ?, in Evian 2015 Workshop on LHC Commissioning, Evian-les-Bains, France, edited by B. Goddard and S. Dubourg (CERn, Geneva, 2015), Report No. CERN-ACC-2015-376.

[51] R. Bruce, C. Bracco, R. De Maria, M. Giovannozzi, A. Mereghetti, D. Mirarchi, S. Redaelli, E. Quaranta, and B. Salvachua, Reaching record-low $\beta^{*}$ at the CERN Large Hadron Collider using a novel scheme of collimator settings and optics, Nucl. Instrum. Methods Phys. Res., Sect. A 848, 19 (2017). 